\title{
Gravitationally Induced Dark Matter Asymmetry and Dark Nucleon Decay
}

\author{
Hooman Davoudiasl* \\ Department of Physics, Brookhaven National Laboratory, Upton, NY 11973, USA
}

\begin{abstract}
The "gravitational baryogenesis" scenario is extended to generate both baryon and dark matter asymmetries, in the matter dominated era corresponding to post-inflationary reheating. A minimal extension requires a singlet fermion $X$ for dark matter and a singlet scalar $S$. With two or more hidden sector fermions, the scenario can lead to nucleon decay into dark matter with a lifetime of order $10^{34-36} \mathrm{yr}$, which is relevant for current or future experiments. The correct multi-component relic density can be obtained if dark matter fermions couple to a sub-GeV vector boson that weakly interacts with the Standard Model through mixing. The typical inflationary scale in the scenario is of order $10^{16} \mathrm{GeV}$ which suggests that tensor mode perturbations could potentially be within observational reach.
\end{abstract}

The cosmological energy densities of baryons and dark matter (DM), respectively denoted by $\Omega_{B}$ and $\Omega_{D M}$, have similar sizes [1, 2]

$$
\frac{\Omega_{D M}}{\Omega_{B}} \approx 5,
$$

even though they have very different properties. This empirical fact provides motivation for postulating a common origin for cosmic baryon and dark matter abundances. A common origin suggests that the DM number density $n_{D M}$, like the number density $n_{B}$ of baryons, is given by an asymmetry. Various cosmological observations imply that the ratio of baryon density to that of entropy $s$ is given by [四

$$
\frac{n_{B}}{s} \approx 10^{-10} .
$$

If the origin of the baryon and DM asymmetries is the same, we may expect $n_{B} \sim n_{D M}$ which implies an asymmetric dark matter (ADM) [3] mass in the $\mathrm{GeV}$ regime. A variety of mechanisms for ADM have been proposed in the literature. See Ref. [4] for some of the pioneering work in this direction and Refs. 3, 0 , for a sample of more recent investigations. Ref. [8] contains some reviews of the subject.

In what follows, we will consider extending the "gravitational baryogenesis" mechanism proposed in Ref. [9] to include the generation of a DM asymmetry. In this scenario, dynamical violation of CPT in an expanding universe leads to the generation of asymmetries, in thermal equilibrium, through the coupling [9]

$$
\frac{1}{M_{c}^{2}} \int d^{4} x \sqrt{-g}\left(\partial_{\mu} \mathcal{R}\right) J_{Q}^{\mu},
$$

where $M_{c}$ is the gravity cutoff scale, $\mathcal{R}$ is the Ricci scalar curvature, and $J_{Q}^{\mu}$ is the current associated with a quantum number $Q$. The scale $M_{c}$ is typically of order the

*email: hooman@bnl.gov reduced Planck mass $\bar{M}_{\mathrm{Pl}} \approx 2.4 \times 10^{18} \mathrm{GeV}$, but could be somewhat different. The universality of gravitational interactions suggests that such couplings generally exist.

We will assume that $Q=B$ is the baryon number in the visible sector. Let $X$ be a Dirac fermion, assumed to be DM, carrying a hidden charge $X=+1$.

Following the arguments presented in Ref. [9], the interaction in Eq. (3) provides a bias in thermal equilibrium that acts as a chemical potential for generating a charge asymmetry. This is due to dynamical CPT violation from the time evolution of the Ricci scalar (this is similar in spirit to "spontaneous baryogenesis" [10]). The baryon asymmetry is then given by [9]

$$
\left.\frac{n_{B}}{s} \approx \frac{\dot{\mathcal{R}}}{M_{c}^{2} T}\right|_{T_{D}},
$$

for $T<T_{D}$, where $T_{D}$ is the temperature at which processes that violate $B$ decouple; a dot represents a time derivative. For $B$ violation, we will consider the $\operatorname{dim}-6$ operator 11.

$$
O_{B X}=\frac{(X u d d)_{R}}{\Lambda^{2}}+\text { H.C. }
$$

where $u$ and $d$ are the up- and down- type quarks in the Standard Model (SM), the subscript $R$ denotes righthanded chirality, and generation and color indices have been suppressed; $\Lambda$ is the scale of $B+X$ violation. Note that $O_{B X}$ preserves $B-X$, which we will assume to be a good symmetry. This assumption excludes neutrino Dirac mass operators of the type $H L X$, where $H$ is the Higgs field and $L$ is a lepton doublet of the SM.

The induced CPT violation from Eq. (3) and the interaction in Eq. (5) lead to the generation of equal asymmetries in $X$ and $B$. The asymmetry in $X$ remains unprocessed. However, the $B$ number can get partially converted into lepton number if the electroweak sphaleron processes are active, corresponding to a reheat temperature $T_{\mathrm{R}} \gtrsim 100 \mathrm{GeV}$, after inflation. For $T_{\mathrm{R}} \gtrsim 100 \mathrm{GeV}$, the well-known results of Ref. [12] then yield

$$
n_{B}=(28 / 79) n_{X}
$$


for the size of the baryon asymmetry at $T \ll 100 \mathrm{GeV}$. Eq. (11) then implies that $m_{X} \approx 2 \mathrm{GeV}$ if the energy density of DM is set by the value of its asymmetry. On the other hand, if $T_{\mathrm{R}} \lesssim 100 \mathrm{GeV}$, the sphalerons are out of thermal equilibrium and the relation $n_{B}=n_{X}$ is maintained down to low temperatures, implying that $m_{X} \approx 5 \mathrm{GeV}$.

In order for the DM density to be set by the asymmetry $n_{X}$, we need the symmetric population of $X$ and $\bar{X}$ particles to annihilate away. As a first attempt, we simply assume that there is a singlet scalar $S$ that couples to $X$ and gives it mass. Let the couplings of $X, S$, and the SM Higgs doublet $H$ be given by

$$
\mathcal{L}=y_{X} S \bar{X} X+\lambda_{S} S^{2} H^{\dagger} H .
$$

If $m_{X}>m_{S}$ the annihilation process $X \bar{X} \rightarrow S S$ could be used for depleting the symmetric $X$ population. Since we are interested in $m_{S} \lesssim 1 \mathrm{GeV}$, we require $\lambda_{S} \lesssim 10^{-5}$ in order to avoid tuned cancelations in the potential for $S$ with $\langle H\rangle \approx 246 \mathrm{GeV}$ in Eq. (7). However, $S$ needs to decay well before Hubble time $t_{H} \sim 1 \mathrm{~s}$, the onset of Big Bang Nucleosynthesis, to avoid large deviations from standard cosmology.

The mass mixing parameter

$$
\mu^{2}=\lambda_{S}\langle H\rangle\langle S\rangle
$$

from the second term in Eq. (7) leads to the mixing of the Higgs $H$ with the singlet $S$, given by the angle

$$
\xi \sim \frac{\mu^{2}}{\langle H\rangle^{2}} .
$$

We will be interested in the case $m_{S} \sim\langle S\rangle \lesssim 1 \mathrm{GeV}$ which implies $\xi \lesssim 10^{-7}$. To estimate an upper bound on the lifetime $\tau_{S}$ of $S$, let us consider the decay into $\mu^{+} \mu^{-}$, via mixing with the Higgs. We then have

$$
1 / \Gamma\left(S \rightarrow \mu^{+} \mu^{-}\right) \approx \frac{16 \pi}{\xi^{2} y_{\mu}^{2} m_{S}} \gtrsim 10^{-2} \mathrm{~s},
$$

where the muon Yukawa coupling is given by $y_{\mu} \approx 5 \times$ $10^{-4}$. The above bound allows for sufficiently fast decay of $S ; \tau_{S} \ll 1 \mathrm{~s}$. However, below we will also consider cases where $S$ may need to be somewhat lighter than $\sim 1 \mathrm{GeV}$ for efficient $X \bar{X}$ annihilation. In that case, without tuning the $S$ mass parameter, $\xi$ would need to be somewhat smaller than $10^{-7}$ which will lead to $\tau_{S} \gtrsim 1 \mathrm{~s}$. We will address this question near the end of this work, by considering annihilation into dark $U(1)_{d}$ vector bosons that kinetically mix with the photon [13].

The cross section for $X \bar{X} \rightarrow S S$ is given by

$$
\sigma(X \bar{X} \rightarrow S S) \sim \frac{y_{X}^{4}}{32 \pi m_{X}^{2}} .
$$

For $m_{X} \approx 2 \mathrm{GeV}$ and $y_{X} \sim 1$, the above equation yields $\sigma_{X \bar{X}} \sim 10^{-3} \mathrm{GeV}^{-2} \sim \mu \mathrm{b}$. We note that this is much larger than a typical thermal relic annihilation cross section $\sim 1 \mathrm{pb}$. Hence, with our typical assumptions, an efficient depletion of the symmetric DM population can be expected in this minimal setup. We will next consider a cosmological context that could lead to sufficient dynamical CPT violation required for the generation of the relic asymmetries.

According to Eq. (1), the value of asymmetry achieved through gravitational genesis depends on cosmological evolution through $\dot{\mathcal{R}}$, which is given by [9]

$$
\dot{\mathcal{R}}=-(1-3 w) \frac{\dot{\rho}}{\bar{M}_{\mathrm{Pl}}^{2}}=\sqrt{3}(1-3 w)(1+w) \frac{\rho^{3 / 2}}{\bar{M}_{\mathrm{Pl}}^{3}},
$$

where $w$ is the ratio of pressure and energy density $\rho$. As an illustrative example, let us consider $w=0$, corresponding to matter domination. Post-inflationary reheating during inflaton oscillations is described by a matter dominated equation of state and hence $w=0$ is a well motivated choice. Using the result derived for this case in Ref. [9] we get

$$
\frac{n_{B}}{s} \approx \frac{T_{D}^{6}}{M_{c}^{2} \bar{M}_{\mathrm{Pl}}^{3} T_{\mathrm{R}}}
$$

which is obtained in a linear approximation, valid for $T_{\mathrm{R}} \gtrsim 10^{-2} T_{D}$. We recall that $T_{D}$ is the temperature at which $B$ violation mediated by the interaction in Eq. (5) becomes decoupled. Assuming $M_{c} \sim \bar{M}_{\mathrm{Pl}}$, one can then obtain the requisite $n_{B} / s$ with $T_{\mathrm{D}} \sim 2 \times 10^{16} \mathrm{GeV}$ and $T_{\mathrm{R}} \sim 10^{16} \mathrm{GeV}$. For these values, one gets $\Lambda \sim$ few $\times$ $10^{16} \mathrm{GeV}$. Here, the energy density at $T=T_{\mathrm{D}}$ is given by $\rho \sim T_{\mathrm{D}}^{8} / T_{\mathrm{R}}^{4}[9]$. Hence, these values of parameters are consistent with an inflationary scale $V_{I}^{1 / 4} \sim 10^{16} \mathrm{GeV}$. Note that much larger inflationary scales may lead to excessive levels of tensor mode perturbations [2, 14, 15].

Since $T_{\mathrm{R}} \gg 100 \mathrm{GeV}$, sphaleron processes will be active at $T<T_{R}$ after a baryon asymmetry has been generated. Hence, baryon number density is given by Eq. (6), implying that $m_{X} \approx 2 \mathrm{GeV}$, for which nucleon decay through the operator in Eq. (5) is not relevant. However, the dark matter particle $X$ is unstable and can decay into a nucleon and a meson with a very long lifetime $\tau_{X} \sim 10^{34} \mathrm{yr}$. The corresponding decay rate of $X$ is well-below what can be detected through astrophysical observations or otherwise. We also note that $S$ - $H$ mixing, without tuning, is typically suppressed by $\xi \lesssim 10^{-7}$ and hence likely unobservable. Thus, it seems that the minimal scenario of gravitationally generated asymmetric dark matter $X$ is largely inaccessible to experimental tests.

The above conclusion can change in an interesting way if ADM is made up of multiple fields. Let us assume that there are $n$ dark fermions $\tilde{X}_{i}, i=1,2, \ldots, n$. One could then generalize Eq. (5) to

$$
O_{B \tilde{X}_{i}}=\sum_{i}^{n} \frac{\left(\tilde{X}_{i} u d d\right)_{R}}{\Lambda_{i}^{2}}+\text { H.C. },
$$


where $\Lambda_{i}$ are scales of $B+X$ violation corresponding to $\tilde{X}_{i}$. If there is a hierarchy among $\Lambda_{i}$, then Eq. (13) suggests that the $B$ violating interaction with the largest $\Lambda_{i}$ will dominate and we are basically back to the minimal model. However, if the ultraviolet model that generates $O_{B \tilde{X}_{i}}$ is flavor symmetric, we then expect $\Lambda_{i}$ to be of similar size. For simplicity, let us focus on a case in which there is such a symmetry for two fields $\tilde{X}_{1}$ and $\tilde{X}_{2}$. In the limit $\Lambda_{1} \approx \Lambda_{2}$, we then get a two component ADM population with $n_{\tilde{X}_{1}} \approx n_{\tilde{X}_{2}}$.

For $\langle S\rangle \neq 0$, Yukawa interactions of the type $y_{i j} S \tilde{X}_{i}^{c} \tilde{X}_{j}$ yield a mass matrix with diagonal entries $m_{1}$ and $m_{2}$ and the off diagonal entries $m_{12}$ and $m_{21}$. We will refer to the mass eigenstates as $X_{1}$ and $X_{2}$, with masses $m_{X_{1}}$ and $m_{X_{2}}$, respectively. We then have $m_{X_{1}}+m_{X_{2}} \approx 4 \mathrm{GeV}$, from the preceding discussion. For $y_{i j} \sim 1$, one could easily have a mass eigenstate that is lighter than a nucleon. For example, with $m_{1}=2.2 \mathrm{GeV}, m_{2}=1.8 \mathrm{GeV}$, and $m_{12}=m_{21}=1.4 \mathrm{GeV}$, we find $m_{X_{1}}=0.6 \mathrm{GeV}$ and $m_{X_{2}}=3.4 \mathrm{GeV}$. Hence, one of the ADM fields, hereafter denoted by $X_{1}$, could be generically lighter than a nucleon without tuning of model parameters.

The above two-component ADM model then turns out to have an interesting signature, namely the decay of protons and neutrons into DM! Such dark nucleon decay (DND) processes are allowed for $m_{X_{1}} \lesssim m_{N}-m_{\pi}$, where $m_{N}$ is the nucleon mass and $m_{\pi}$ is the mass of the pion. Note that within the inflationary reheating scenario (i.e. with $w=0$ ) discussed above, we end up with scales $\Lambda_{1,2} \gtrsim 10^{16} \mathrm{GeV}$, close to typical Grand Unified Theory (GUT) scales. This suggests that the associated DND rates would be in a range accessible to current or future experiments [16]. These estimates were obtained assuming $M_{c} \sim \bar{M}_{\mathrm{Pl}}$. However, (quantum) gravity effects at very high scales could yield a range of values for $M_{c}$. Hence, one may assume $M_{c}=\kappa \bar{M}_{\mathrm{Pl}}$, with $\kappa \sim 0.05-1$, at the level of our effective theory treatment. In that case, Eqs. (5) and (13) imply nucleon lifetimes of order $10^{34-36}$ yr can result from dark decays.

The DND discussed above is not entirely "dark" as it includes a meson: $N \rightarrow X_{1} \pi, X_{1} K, \ldots$. Such processes will mimic standard nucleon decay (SND) into a neutrino and a meson, $N \rightarrow \nu \pi, \nu K, \ldots$, predicted in other contexts, like GUT scenarios. However, for DND the meson kinematics are expected to be different from that of the SND involving a neutrino. (This is reminiscent of annihilation of nucleons in scattering from ADM, as discussed in Refs. [7, 17.) The meson momenta for $N \rightarrow \nu \pi(K)$ and $N \rightarrow X_{1} \pi(K)$ could differ significantly, depending on the value of $m_{X_{1}}$. As expected, the lighter the DM particle $X_{1}$, the more similar DND and SND are. For instance, the SND meson momenta are given by $p_{\pi(K)}^{\mathrm{SND}} \approx 460(340) \mathrm{MeV}$ (see Table 四), whereas with $m_{X_{1}}=300 \mathrm{MeV}$ we have $p_{\pi(K)}^{\text {DND }} \approx 410(245) \mathrm{MeV}$. However, if $m_{X_{1}}=600 \mathrm{MeV}$, we find $p_{\pi}^{\text {DND }} \approx 250 \mathrm{MeV}$ and

\begin{tabular}{|c|c|c|}
\hline Decay Channel & $p_{\text {meson }}^{\text {SND }}(\mathrm{MeV})$ & $\left(p_{\text {meson }}^{\text {DND }}, m_{X_{1}}\right)(\mathrm{MeV})$ \\
\hline$N \rightarrow \pi$ & 460 & $\begin{array}{c}(410,300) \\
(250,600)\end{array}$ \\
\hline$N \rightarrow K$ & 340 & $\begin{array}{c}(245,300) \\
(-, 600)\end{array}$ \\
\hline
\end{tabular}

TABLE I: Sample meson momenta in standard and dark nucleon decays, for $m_{X_{1}}=300,600 \mathrm{MeV}$.

the kaon mode is forbidden. Thus, for somewhat heavier $X_{1}$, we find markedly different pion kinematics.

As discussed in Ref. [17], kinematic differences can help distinguish novel decay channels such as DND. On the other hand, applying the existing nucleon lifetime constraints to the new physics must be done with care, as kinematics can affect the detection efficiency. For example, if $m_{X_{1}}$ is sufficiently close to the kinematic limit for allowed decays, the pion can be very slow and below Cerenkov threshold. On the other hand, in case of neutral meson decays into diphotons, the suppressed boost could help in resolving the photon pair and event reconstruction.

Here, we would like to add that there are other cosmological epochs governed by $w \neq 0$ in which one could consider the interaction (3) 91. For example, using the results of Ref. [9], it seems that $w \sim 3 / 4$ and $\Lambda \sim$ few $\times 10^{12} \mathrm{GeV}$ could yield $n_{B} / s \sim 10^{-10}$. For values of $\Lambda$ in this range, $\tau_{X} \sim 10^{27} \mathrm{~s}$ is near the current observational bounds [18] for $m_{X} \sim 2 \mathrm{GeV}$ (DND irrelevant) and could lead to an indirect DM decay signal. However, the post-inflationary reheating characterized by $w=0$, considered above, is well-motivated and can be a generic feature of standard cosmological scenarios. We expect that our main conclusions can be accommodated by various conventional inflationary models. We also note that since sufficient asymmetry for $w=0$ requires inflationary scales $V_{I}^{1 / 4} \gtrsim 10^{16} \mathrm{GeV}$, this scenario would typically suggest that the detection of tensor mode perturbations could be within observational reach [2].

Before closing, we will examine a variant scenario that includes a light vector boson $Z_{d}$ associated with a dark $U(1)_{d}$ force that mediates $X \bar{X}$ annihilation [19]. Here, $Z_{d}$ couples to $X$ and kinetically mixes with the photon; such a vector is often referred to as a "dark photon" 20]. This setup avoids potential problems with a long-lived $S$ when $m_{X}<1 \mathrm{GeV}$, as in the interesting case with multiple ADM fermions. For operators of the type (5) to be allowed, we have to assume that $X_{R}$ is not charged under $U(1)_{d}$. However, $X_{L}$ can have the required dark gauge charge. To allow a Yukawa coupling of the type $y_{X} S \bar{X}_{L} X_{R}$, the scalar $S$ needs to be charged under the dark force. This will also ensure that $Z_{d}$ will have a nonzero mass $m_{Z_{d}} \sim g_{d}\langle S\rangle$, where $g_{d}$ is the $U(1)_{d}$ gauge coupling. For $m_{Z_{d}} \ll m_{X}$, the annihilation process 
$X \bar{X} \rightarrow Z_{d} Z_{d}$ has a cross section

$$
\sigma\left(X \bar{X} \rightarrow Z_{d} Z_{d}\right) v \sim \frac{g_{d}^{2} y_{X}^{2}}{32 \pi m_{X}^{2}},
$$

where $v$ is relative velocity. For instance, with $g_{d} \sim$ $y_{X} / 10 \sim 0.1$ and $m_{X} \sim 1 \mathrm{GeV}$, we find an annihilation cross section $\sim 40 \mathrm{nb}$ which is quite sufficient for removing the symmetric $X$ density.

The lifetime of $Z_{d}$ depends on the degree of kinetic mixing with the photon parameterized by $\varepsilon$. For $m_{Z_{d}} \sim 100 \mathrm{MeV}$, experimental bounds require $\varepsilon \lesssim$ few $\times 10^{-3}$ (for a summary of recent constraints see, e.g., Refs. 22, 23]). However, to avoid conflict with direct detection bounds for $m_{X_{2}} \sim$ few $\mathrm{GeV}$, we may need to consider $\varepsilon \lesssim 10^{-5}$ ]. For these values, $Z_{d}$ lifetime is of order $16 \pi /\left(\varepsilon^{2} m_{Z_{d}}\right) \gtrsim 10^{-11} \mathrm{~s}$ which does not pose a difficulty. Here, we have assumed that the decays of $Z_{d}$ are dominated by the visible $e^{+} e^{-}$channel. This is consistent with our requirement $m_{X}>m_{Z_{d}}$ (see Refs. 24 26 for a discussion of $Z_{d}$ phenomenology when dark matter final states are dominant).

In summary, we extended "gravitational baryogenesis" to accommodate the generation of a dark matter asymmetry. We focused on the well-motivated matter dominated cosmological equation of state $(w=0)$ that characterizes reheating through inflaton oscillations. The minimal required extension can be implemented by adding a singlet Dirac fermion dark matter and a singlet scalar. This setup leads to an unstable dark matter with a lifetime of order $10^{34} \mathrm{yr}$. The minimal model (for $w=0$ ) does not offer readily accessible observational signatures. However, a modestly enlarged dark sector with more than one fermion could lead to the interesting possibility of nucleon decays into dark matter fermions. We showed, as an example, that a simple two-fermion dark matter sector could lead to dark nucleon decays, with inverse rates $10^{34-36} \mathrm{yr}$, relevant to current or future experiments. In our reference $w=0$ cosmology, the large inflationary scale required for sufficient dark matter and baryon asymmetries typically suggests that tensor mode perturbations may be within reach of astrophysical measurements.

We thank R. Kitano for collaboration during the early stages of this work. This work is supported in part by the United States Department of Energy under Grant Contracts DE-AC02-98CH10886.

[1] J. Beringer et al. [Particle Data Group Collaboration], Phys. Rev. D 86, 010001 (2012).

[2] P. A. R. Ade et al. [Planck Collaboration], arXiv:1303.5076 [astro-ph.CO].

[3] D. E. Kaplan, M. A. Luty and K. M. Zurek, Phys. Rev. D 79, 115016 (2009) [arXiv:0901.4117 [hep-ph]].
[4] P. Hut and K. A. Olive, Phys. Lett. B 87, 144 (1979); S. Nussinov, Phys. Lett. B 165, 55 (1985); S. Dodelson and L. M. Widrow, Phys. Rev. Lett. 64, 340 (1990); S. M. Barr, R. S. Chivukula and E. Farhi, Phys. Lett. B 241, 387 (1990); D. B. Kaplan, Phys. Rev. Lett. 68, 741 (1992).

[5] R. Kitano and I. Low, Phys. Rev. D 71, 023510 (2005) [hep-ph/0411133]; G. R. Farrar and G. Zaharijas, Phys. Rev. Lett. 96, 041302 (2006) [hep-ph/0510079]; M. Y. .Khlopov, Pisma Zh. Eksp. Teor. Fiz. 83, 3 (2006) [JETP Lett. 83, 1 (2006)] [astro-ph/0511796]; P. -H. Gu, Phys. Lett. B 657, 103 (2007) [arXiv:0706.1946 [hep-ph]]; H. An, S. -L. Chen, R. N. Mohapatra and Y. Zhang, JHEP 1003, 124 (2010) [arXiv:0911.4463 [hep-ph]]; N. Haba and S. Matsumoto, Prog. Theor. Phys. 125, 1311 (2011) [arXiv:1008.2487 [hep-ph]]; M. R. Buckley and L. Randall, JHEP 1109, 009 (2011) [arXiv:1009.0270 [hep-ph]].

[6] J. Shelton and K. M. Zurek, Phys. Rev. D 82, 123512 (2010) [arXiv:1008.1997 [hep-ph]].

[7] H. Davoudiasl, D. E. Morrissey, K. Sigurdson and S. Tulin, Phys. Rev. Lett. 105, 211304 (2010) [arXiv:1008.2399 [hep-ph]].

[8] H. Davoudiasl and R. N. Mohapatra, New J. Phys. 14, 095011 (2012) [arXiv:1203.1247 [hep-ph]]; K. Petraki and R. R. Volkas, Int. J. Mod. Phys. A 28, 1330028 (2013) [arXiv:1305.4939 [hep-ph]]; K. M. Zurek, arXiv:1308.0338 [hep-ph].

[9] H. Davoudiasl, R. Kitano, G. D. Kribs, H. Murayama and P. J. Steinhardt, Phys. Rev. Lett. 93, 201301 (2004) [arXiv:hep-ph/0403019].

[10] A. G. Cohen and D. B. Kaplan, Phys. Lett. B 199, 251 (1987).

[11] This type of operator has also been considered in other ADM models; see for example Refs. 13, 6, 7].

[12] J. A. Harvey and M. S. Turner, Phys. Rev. D 42, 3344 (1990).

[13] B. Holdom, Phys. Lett. B 166, 196 (1986).

[14] A. D. Linde, Phys. Lett. B 129, 177 (1983).

[15] D. H. Lyth, Phys. Lett. B 147, 403 (1984) [Erratum-ibid. B 150, 465 (1985)].

[16] K. Kobayashi et al. [Super-Kamiokande Collaboration], Phys. Rev. D 72, 052007 (2005) [hep-ex/0502026]; Y. Suzuki et al. [TITAND Working Group Collaboration], hep-ex/0110005; M. V. Diwan, R. L. Hahn, W. Marciano, B. Viren, R. Svoboda, W. Frati, K. Lande and A. K. Mann et al., hep-ex/0306053; A. Bueno, Z. Dai, Y. Ge, M. Laffranchi, A. J. Melgarejo, A. Meregaglia, S. Navas and A. Rubbia, JHEP 0704, 041 (2007) [hep-ph/0701101].

[17] H. Davoudiasl, D. E. Morrissey, K. Sigurdson and S. Tulin, Phys. Rev. D 84, 096008 (2011) [arXiv:1106.4320 [hep-ph]].

[18] N. F. Bell, A. J. Galea and K. Petraki, Phys. Rev. D 82, 023514 (2010) [arXiv:1004.1008 [astro-ph.HE]].

[19] M. Pospelov, A. Ritz and M. B. Voloshin, Phys. Lett. B 662, 53 (2008) [arXiv:0711.4866 [hep-ph]].

[20] An extension of the dark photon scenario can include mass mixing with the SM $Z$ boson, which allows $Z_{d}$ to behave as a "dark $Z$ " as explained in Ref. 21. For simplicity, we only focus on the dark photon possibility, though the assumed $Z_{d}$ could also have dark $Z$ properties.

[21] H. Davoudiasl, H. -S. Lee and W. J. Marciano, Phys. Rev. D 85, 115019 (2012) [arXiv:1203.2947 [hep-ph]]. 
[22] H. Davoudiasl, H. -S. Lee, I. Lewis and W. J. Marciano, Phys. Rev. D 88, 015022 (2013) [arXiv:1304.4935 [hep$\mathrm{ph}]$.

[23] S. Andreas, M. D. Goodsell and A. Ringwald, arXiv:1306.1168 [hep-ph].

[24] B. Batell, M. Pospelov and A. Ritz, Phys. Rev. D 80,
095024 (2009) [arXiv:0906.5614 [hep-ph]].

[25] P. deNiverville, M. Pospelov and A. Ritz, Phys. Rev. D 84, 075020 (2011) [arXiv:1107.4580 [hep-ph]].

[26] E. Izaguirre, G. Krnjaic, P. Schuster and N. Toro, arXiv:1307.6554 [hep-ph]. 